\title{
Induced Representations, Reproducing Kernels and the Conformal Group
}

\author{
A. L. Carey \\ Department of Mathematical Physics, University of Adelaide, Adelaide, South Australia 5001
}

\begin{abstract}
The properties of "induced" (or multiplier) representations of groups which act in Hilbert spaces with a reproducing kernel are investigated. A resumé of earlier work is followed by a discussion of criteria for the irreducibility of such representations. The notions of reproducing kernel and positive definite spherical function are found to overlap. As a result, functional equations (analogous to those of Godement for spherical functions) are found for the reproducing kernel. The abstract theory is illustrated by certain discrete series representations of the conformal group and by their "limit points". In particular the so-called ladder representations (which give rise to the conformal symmetry of zero mass particles) are analysed from this viewpoint.
\end{abstract}

\section{Reproducing Kernels}

\section{Introduction}

This paper is mainly concerned with developing some results on group representations in Hilbert spaces with reproducing kernels. The ideas were motivated by the papers of Ruhl [2] on the conformal group, Bargmann's basic work [3] on the commutation relations and the paper of Perelomov [4] in which he points out that the notion of "coherent state" and reproducing kernel are the same.

The theory has its origins in the papers of Krein [9] and Aronszajn [10] although the foundations for the ideas developed here were laid by Kunze [1]. Subsection 2.1 is devoted to a resume of the results of [1]. This is followed by a discussion of criteria for the irreducibility of "induced representations" in reproducing kernel Hilbert spaces. The rest of Section 2 is concerned with the relationship between spherical functions and reproducing kernels. The existence of functional equations for reproducing kernels and their relationship to the functional equations satisfied by spherical functions of height one (see Godement [5] for this terminology) is investigated.

The general theory is illustrated by using the work of Graev [6] and Ruhl [2] on the discrete series representations of the conformal group [which for our 
purposes will be $\mathrm{SU}(2,2)]$. (Note that it was observed by Langlands [7] that the holomorphic discrete series representations of a semisimple Lie group can be constructed in Hilbert spaces with reproducing kernels.) In particular, we consider a series of representations labelled by a positive integer $n$ which, for $n \geqq 4$, lie in the discrete series but for $n<4$ may be regarded as "limit points". An example of physical interest is the case $n=1$ which corresponds to the zero mass, zero helicity representation of the conformal group. This leads us to consider the construction of the so-called "ladder representations" of SU $(2,2)[8]$ in Hilbert spaces of functions holomorphic in the field theoretic "future tube". The reproducing kernels for these spaces are exhibited and the relationship to the usual infinitesimal form of the ladder representations [8] is determined by the fact that the Minkowski space wave functions are the boundary values in a "limit in mean" sense, of the holomorphic functions. Connections are made with the classical formulae for solutions of the wave equation.

\section{General Theory}

\subsection{The Work of Kunze}

In this subsection I will review the results of [1] and introduce the basic definitions and notation. ${ }^{1}$

Definition 2.1.1. Let $X$ be a locally compact Hausdorff space, $V$ a complex Hilbert space and $H$ a Hilbert space whose elements are functions from $X$ to $V$ which are continuous in the norm topology on $V$. Suppose there exists a function $\phi: X \times X \rightarrow B(V)$ (the bounded operators on $V$ ) such that

(i) the functions

$$
y \rightarrow \phi_{x}^{*} v(y) \stackrel{\text { def }}{=} \phi(x, y) * v, \quad v \in V .
$$

lie in $H$ for all $x \in X$,

(ii) $\left\langle\phi_{x}^{*} v, F\right\rangle_{H}=\langle v, F(x)\rangle_{V}$

for all $F \in H$ and $v \in V$, then $H$ is called a reproducing kernel Hilbert space with kernel $\phi$.

Remarks. 1. The functions $\phi_{x}^{*} v$ are the "coherent states".

2. We use the adjoint $\phi(x, y)^{*}$ of $\phi(x, y)$ in the definition to accord with the notation of the examples of Section 3.

Let $G$ be a locally compact group with $X$ of the form $G / K, K$ some closed subgroup of $G$.

Definition 2.1.2. A strongly continuous function $\tau: X \times G \rightarrow B(V)$ is a cocycle if

(i) $\tau(x, e)=I(I$, the identity operator and $e$ the identity of $G)$,

(ii) $\tau\left(x, g_{1} g_{2}\right)=\tau\left(x, g_{1}\right) \tau\left(g_{1}^{-1} \cdot x, g_{2}\right)$.

If $K$ fixes the point $x_{0}$ say, in $X$, then (i) and (ii) imply that $k \rightarrow \tau\left(x_{0}, k\right)$ is a representation of $K$. Call this representation $\sigma$.

1 Note that our notation and formulation differs in several important respects from that used in [1] 
If $H$ is a Hilbert space whose elements are functions from $X$ to $V$ such that the equation

$$
\left(U_{g} F\right)(x)=\tau(x, g) F\left(g^{-1} \cdot x\right)
$$

defines $g \rightarrow U_{g}$ as a strongly continuous unitary representation of $G$ then we call $U$ the representation induced by the cocycle $\tau$ and write $\operatorname{ind}_{\tau}(H)$. This definition can be seen to be equivalent to the usual one (Varadarajan [10]) in certain circumstances. To see this define $b: X \rightarrow B(V)$ by

$$
b(x)=\tau\left(x_{0}, \gamma(x)^{-1}\right)
$$

where $\gamma: X \rightarrow G$ is a regular Borel cross-section [10] of $G \rightarrow G / K$ such that $\gamma(x) \cdot x_{0}=x$ and $\gamma\left(x_{0}\right)=e$. Now define

$$
(B F)(x)=b(x) F(x) \text {. }
$$

Then provided $B H \leqq L^{2}(X, V, \mu)$ where $\mu$ is an invariant measure on $X$, it is not difficult to show that $B$ intertwines $\operatorname{ind}_{\tau}(H)$ with the representation induced by the cocycle

$$
\sigma_{\gamma}(x, g)=\tau\left(x_{0}, \gamma(x)^{-1} g \gamma\left(g^{-1} \cdot x\right)\right) .
$$

Hence whenever $\sigma_{\gamma}(x, g)$ is unitary for all $x, g$ then $B$ sets up an equivalence of $\operatorname{ind}_{\tau}(H)$ with a subrepresentation of the representation of $G$ on $L^{2}(X, V, \mu)$ induced by $\sigma$. In general however, this is not the case. Examples of such representations will be considered in Sections 3 and 4.

The first result of $[1]$ is as follows.

Theorem 2.1.3 (Kunze). If $H$ is a Hilbert space (whose elements are functions from $X$ to $V$ ) having reproducing kernel $\phi$, then the formula (2.1) defines a strongly continuous unitary representation of $G$ if and only if $\phi$ satisfies the covariance relation

$$
\phi\left(g^{-1} \cdot x, y\right)^{*} \tau(x, g)^{*}=\tau\left(y, g^{-1}\right) \phi(x, g \cdot y)^{*} .
$$

Now, the kernel $\phi$ on the homogeneous space $X$ may be "lifted" to a kernel on the group $G$. Following [1] define:

$$
\chi\left(g_{1}, g_{2}\right)^{*}=\tau\left(x_{0}, g_{2}^{-1}\right) \phi(x, y)^{*} \tau\left(x_{0}, g_{1}^{-1}\right)^{*}
$$

where $g_{1} \cdot x_{0}=x$ and $g_{2} \cdot x_{0}=y$. Then it is straightforward to verify

(i) $\chi\left(g_{1}, g_{2}\right)^{*}=\chi\left(g_{2}, g_{1}\right)$, [note that by definition $\left.\phi(x, y)^{*}=\phi(y, x)\right]$,

(ii) $\chi\left(g g_{1}, g g_{2}\right)^{*}=\chi\left(g_{1}, g_{2}\right)^{*}$,

(iii) $\chi\left(g_{1} k_{1}, g_{2} k_{2}\right)^{*}=\tau\left(x_{0}, k_{2}^{-1}\right) \chi\left(g_{1}, g_{2}\right)^{*} \tau\left(x_{0}, k_{1}^{-1}\right)^{*}$.

We can also "lift" the Hilbert space $H$ by defining for each $F \in H$ a $V$-valued function on $G$ by

$$
F^{\sim}(g)=\tau\left(x_{0}, g^{-1}\right) F\left(g \cdot x_{0}\right) .
$$

The functions $F^{\sim}$ form a Hilbert space $H^{\sim}$ with inner product

$$
\left\langle F^{\sim}, F^{\sim \prime}\right\rangle \stackrel{\text { def }}{=}\left\langle F, F^{\prime}\right\rangle_{H} .
$$


Hence,

$$
\begin{aligned}
\left\langle\chi_{g}{ }^{*} v, F^{\sim}\right\rangle & =\left\langle\phi_{g \cdot x_{0}}^{*} \tau\left(x_{0}, g^{-1}\right)^{*} v, F\right\rangle_{H} \\
& =\left\langle v, \tau\left(x_{0}, g^{-1}\right) F\left(g \cdot x_{0}\right)\right\rangle_{V} \\
& =\left\langle v, F^{\sim}(g)\right\rangle_{V} .
\end{aligned}
$$

So $H^{\top}$ has reproducing kernel $\chi$ and carries a representation $W$ defined by

$$
\left(W_{g} F^{\sim}\right)\left(g_{0}\right)=F^{\sim}\left(g^{-1} g_{0}\right)
$$

which is equivalent to $\operatorname{ind}_{\tau}(H)$.

Let us assume that $k \rightarrow \tau\left(x_{0}, k\right)$ is unitary. Let $H$ be as above. If we make the further restriction that the maps $E_{x}: f \rightarrow f(x)$ from $H$ to $V$ have dense range for all $x \in X$, then Kunze has characterized those group representations which may be constructed in a reproducing kernel Hilbert space. (Note however that there exist reproducing kernel Hilbert spaces which do not satisfy this restriction, cf. Section 4.)

Definition 2.1.4 (Kunze). Let $U$ be a continuous unitary representation of $G$ on a Hilbert space $H_{0}, K$ a closed subgroup of $G$ and $\sigma$ a strongly continuous unitary representation of $K$ on a Hilbert space $V$. If there exists a closed subspace $V^{\prime}$ of $H_{0}$ which carries a representation of $K$ unitarily equivalent to $\sigma$ and is such that the linear span of $\left\{U_{g} v \mid g \in G, v \in V^{\prime}\right\}$ is dense in $H_{0}$, then we say that $U$ is of type $\sigma$.

Given a representation $U$ of type $\sigma$, and $A: V \rightarrow V^{\prime}$ a unitary transformation setting up the equivalence of the preceding definition, define $\Phi: G \rightarrow B(V)$ by

$$
\langle u, \Phi(g) v\rangle=\left\langle A u, U_{g} A v\right\rangle, \quad u, v \in V .
$$

Now, defining $\chi\left(g_{1}, g_{2}\right)^{*}=\Phi\left(g_{1}^{-1} g_{2}\right)^{*}$ Kunze shows (Theorem 5 of [1]) that there is a reproducing kernel Hilbert space $H^{\sim}$ (with kernel $\chi$ ) consisting of continuous functions from $G$ to $V$, such that $\chi$ satisfies

$$
\chi\left(g_{1} k_{1}, g_{2} k_{2}\right)^{*}=\sigma\left(k_{2}^{-1}\right) \chi\left(g_{1}, g_{2}\right)^{*} \sigma\left(k_{1}\right) .
$$

Furthermore, the representation of $G$ defined by

$$
(g \cdot F)\left(g_{0}\right)=F\left(g^{-1} g_{0}\right)\left(F \in H^{\Upsilon}\right)
$$

is equivalent to $U$.

The above discussion establishes the main results of [1]. The remainder of this section is concerned with developing some elementary consequences of the above definitions. Note that the assumption that $E_{x}$ has dense range for all $x \in X$ will not be made (this leads to a more cumbersome notation than that adopted by Kunze).

(i) Let $H$ be as above and $H_{0}$ a subspace of $H$. Then $H_{0}$ has a reproducing kernel $\psi: X \times X \rightarrow B(V)$ defined by

$$
\left\langle\psi(x, y)^{*} u, v\right\rangle_{V}=\left\langle P \phi_{x}^{*} u(y), v\right\rangle_{V}
$$

where $P$ is the orthogonal projection from $H$ to $H_{0}$. 
(ii) If we drop the continuity requirement from the definition of $H$ then it can be shown that a Hilbert space of functions from $X$ to $V$ has a reproducing kernel if and only if every element $F$ of the space satisfies

$$
\|F(x)\|_{V} \leqq \omega(x)\|F\|_{H} \text { for all } x \in X,
$$

where $\omega(x)>0$ depends only on $x$. (This requires some work to prove.)

(iii) Quite often $H$ will be a subspace of a larger Hilbert space $H_{1}$. Then $\phi$ defines the orthogonal projection onto $H_{1}$ by $f \rightarrow F$ where

$\langle v, F(x)\rangle_{V}=\left\langle\phi_{x}{ }^{*} v, f\right\rangle_{H_{1}}, \quad f \in H_{1}$.

(iv) By the Cauchy-Schwarz inequality we have the useful bounding property of $\phi$ :

$$
\left|\langle v, F(x)\rangle_{V}\right| \leqq\langle v, \phi(x, x) v\rangle_{V}^{1 / 2}\|F\|_{H}
$$

for all $v \in V$.

For the remainder of the paper we will assume that $k \rightarrow \tau\left(x_{0}, k\right)$ is unitary, that $\phi\left(x_{0}, x_{0}\right)=I$ and that $H$ carries the unitary representation $\operatorname{ind}_{\tau}(H)$ of $G$. With these assumptions we prove (cf. Theorem 4 of [1]):

Lemma 2.1.5. Denote the restriction of $\operatorname{ind}_{\tau}(H)$ to $K$ by $k \rightarrow S_{k}$. Then the vectors $\left\{\phi_{x_{0}}{ }^{*} v \mid v \in V\right\}$ span a subspace $V_{0}$ of $H$ carrying a representation of $K$ equivalent to $\sigma$. Further, the projection $P_{0}$ onto this subspace is given by

$$
\begin{aligned}
P_{0}\left(\phi_{x}{ }^{*} u\right)(y) & =\phi\left(x_{0}, y\right)^{*} \phi\left(x, x_{0}\right)^{*} u \\
& \equiv \phi_{x_{0}}{ }^{*} \phi\left(x, x_{0}\right)^{*} u(y) .
\end{aligned}
$$

Proof. By virtue of (2.2),

$$
\begin{aligned}
S_{k}\left(\phi_{x_{0}}{ }^{*} u\right)(x) & =\tau(x, k) \phi_{x_{0}}{ }^{*} u\left(k^{-1} \cdot x\right) \\
& =\phi_{x_{0}}{ }^{*} \tau\left(x_{0}, k^{-1}\right)^{*} u(x) .
\end{aligned}
$$

Hence $S_{k} \phi_{x_{0}}{ }^{*} u=\phi_{x_{0}}{ }^{*} \sigma(k) u$. Clearly therefore, $S$ acting in the space $V_{0}$ gives a representation equivalent to $\sigma$ (note that the map $V \rightarrow V_{0}$ defined by

$$
v \rightarrow \phi_{x_{0}}{ }^{*} v
$$

is an isometry).

Consider the operator $P_{0}$ defined on the vectors $\phi_{x}{ }^{*} u$ by (2.13). Observe that the space $H_{\phi}$ defined as the linear span of $\left\{\phi_{x}^{*} v \mid v \in V, x \in X\right\}$ is dense in $H$. So extending $P_{0}$ linearly to $H_{\phi}$ makes $P_{0}$ densely defined. Further, if

$$
F=\sum_{x, u} \lambda_{x} \phi_{x}^{*} u
$$

is an element of $H_{\phi}$, then by direct computation

$$
\left\|P_{0} F\right\|^{2}=\left\langle F\left(x_{0}\right), F\left(x_{0}\right)\right\rangle_{V} .
$$

But by (2.12) we have

$$
\left\|F\left(x_{0}\right)\right\| \leqq\|F\| \text {. }
$$

So $P_{0}$ is bounded on $H_{\phi}$ and hence on $H$. As $\phi\left(x_{0}, x_{0}\right)=I$ so $P_{0}^{2}=P_{0}$. It is straightforward to check that $P_{0}=P_{0}{ }^{*}$ thus completing the proof. 
Remark. Acting on an arbitrary $F \in H, P_{0}$ has the form

$$
\left(P_{0} F\right)(x)=\phi\left(x_{0}, x\right)^{*} F\left(x_{0}\right) \text {. }
$$

\subsection{Criteria for Irreducibility}

Following Krein [9] we introduce the

Definition 2.2.1. Let $H, \phi$ be as for Lemma 2.1.5 and denote the strongly continuous $V$-valued functions on $X$ by $C(X, V)$. Call $\phi(G, K, V)$-zonal if the conditions:

(i) there is a function $\psi: X \times X \rightarrow B(V)$ such that both $\psi$ and $\phi-\psi$ are reproducing kernels for Hilbert spaces of functions contained in $C(X, V)$,

(ii) $\psi$ satisfies (2.2), force $\psi$ to be a multiple of $\phi$.

Note that whenever $\psi$ satisfies (i) we can define

$$
A\left(\phi_{x}^{*} u, \phi_{y}{ }^{*} v\right)=\left\langle\psi(x, y)^{*} u, v\right\rangle_{V} .
$$

$A$ can be extended to a sesquilinear functional on $H_{\phi} \times H_{\phi}\left(H_{\phi}\right.$ as in the proof of Lemma 2.1.5). Since $\phi-\psi$ is also a reproducing kernel we have

$$
A(F, F) \leqq\langle F, F\rangle_{H}
$$

for all $F \in H_{\phi}$. Thus $A$ is continuous from $H_{\phi} \times H_{\phi}$ to $\mathbb{C}$ and therefore extends uniquely to $H \times H$. By the Riesz representation theorem there is a bounded (self-adjoint) operator $A^{\sim}: H \rightarrow H$ such that

$$
\left\langle A^{\sim} F, F^{\prime}\right\rangle=A\left(F, F^{\prime}\right)
$$

for all $F, F^{\prime} \in H$.

Now let us record the

Lemma 2.2.2. If $B: H \rightarrow H$ is bounded then $B$ has a kernel $b: X \times X \rightarrow B(V)$ such that

$$
\langle u,(B F)(x)\rangle_{V}=\left\langle b_{x} u, F\right\rangle_{H}
$$

where $b_{x}(y)=b(x, y)$ is defined $b y$

$$
\langle b(x, y) u, v\rangle_{V}=\left\langle B^{*} \phi_{x}^{*} u(y), v\right\rangle_{V} .
$$

Further, $B$ is in the commuting algebra of $\operatorname{ind}_{\tau}(H)$ if and only if $b$ satisfies (2.2). lies in

The proof is straightforward so we omit it. The point of the preceding discussion

Lemma 2.2.3. $\phi$ is $(G, K, V)$-zonal if and only if $\operatorname{ind}_{\tau}(H)$ is irreducible.

Proof. Suppose $\operatorname{ind}_{\tau}(H)$ is not irreducible and $P$ is a non-zero projection onto a proper invariant subspace. Now $P H$ has a reproducing kernel say $\psi$ and hence the kernel for $(I-P) H$ is $\phi-\psi$. Now applying Lemma 2.2.2 to $P$ forces $\psi$ to satisfy conditions (i) and (ii) of Definition 2.2.1. So $\phi$ is not zonal.

Conversely, let $\psi$ satisfy conditions (i) and (ii) of the definition with $\psi$ not a multiple of $\phi$. By the remarks preceding Lemma 2.2.2, $\psi$ defines an operator $A^{\sim}: H \rightarrow H$. Now by Lemma 2.2.2 $A^{\sim}$ is in the commuting algebra of ind ${ }_{\tau}(H)$ noting 
that $A^{\sim}$ necessarily has as its kernel, $\psi$ ). Since $\psi$ is not a multiple of $\phi$, this forces the commuting algebra to be non-trivial. Equivalently, $\operatorname{ind}_{\tau}(H)$ is not irreducible.

Our object now is to determine conditions under which a given kernel is zonal. As before denote the restriction of $\operatorname{ind}_{\tau}(H)$ to $K$ by $k \rightarrow S_{k}$.

Theorem 2.2.4. Let $H, \phi, \operatorname{ind}_{\tau}(H)$ be as above and $P_{0}$ be the projection defined in Lemma 2.1.5. Suppose that

$$
k \rightarrow \tau\left(x_{0}, k\right)
$$

is an irreducible representation of $K$ and there exists a subgroup $G_{0}$ of $G$ such that the following condition holds: there is a projection $P_{c}$ with $P_{0} \geqq P_{c}$, lying in the centre of the commuting algebra of the representation $\operatorname{ind}_{\tau}(H)$ restricted to $G_{0}$. Then $\phi$ is $(G, K, V)$-zonal.

Remark. One way in which the condition on $P_{c}$ may be realized is if there is a subspace $P_{c} H$ of $P_{0} H$, such that on restricting ind ${ }_{\tau}(H)$ to $G_{0}$, one obtains a maximal primary (or factor) representation of $G_{0}$ (in the terminology of Mackey [11]) in $P_{c} H$.

Proof. Suppose that $\operatorname{ind}_{\tau}(H)$ is not irreducible and that $P$ is the projection onto a proper invariant subspace. Define the kernel $\psi$ for $P H$ by (2.10). From the hypotheses on $P_{c}$ we deduce that $P_{c} P_{0}=P_{0} P_{c}$ and $P_{c} P=P P_{c}$. Further, the map $v \rightarrow \phi_{x_{0}}{ }^{*} v$ is an isometry from $V$ onto $P_{0} H$ and therefore $P_{c}$ defines a projection $P_{c}^{\sim}$ say, on $V$. Hence, for $u \in P_{c}^{\sim} V$ :

$$
\psi_{x_{0}}{ }^{*} u=P \phi_{x_{0}}{ }^{*} u=P P_{c} \phi_{x_{0}}{ }^{*} u=P_{c} \psi_{x_{0}}{ }^{*} u \text {. }
$$

Further $\psi$ satisfies (2.2) and hence the relation

$$
\psi\left(x_{0}, x_{0}\right)=\tau\left(x_{0}, k^{-1}\right) \psi\left(x_{0}, x_{0}\right) \tau\left(x_{0}, k\right) .
$$

But $k \rightarrow \tau\left(x_{0}, k\right)=\sigma(k)$ is irreducible and so $\psi\left(x_{0}, x_{0}\right)$ is a multiple: $\lambda I$ of the identity. Now (2.14) implies that $\psi_{x_{0}}{ }^{*} u$ lies in $P_{0} H$ and hence that

$$
\left\langle\phi_{x_{0}}{ }^{*} v, \psi_{x_{0}}{ }^{*} u\right\rangle_{H}=\lambda\langle v, u\rangle_{V}
$$

for all $v \in V$. From this it follows readily that $\psi_{x_{0}}{ }^{*} u=\lambda \phi_{x_{0}}{ }^{*} u$ for all $u \in P_{c} V$. This forces $\lambda=1$ and since $\sigma$ is irreducible we must have

$$
\psi\left(x_{0}, x\right)^{*}=\phi\left(x_{0}, x\right)^{*} .
$$

Now let $g \cdot x_{0}=x$ so that from (2.2)

$$
\begin{aligned}
\phi(x, y)^{*} \tau\left(x_{0}, g^{-1}\right)^{*} & =\tau(y, g) \phi\left(x_{0}, g^{-1} \cdot y\right)^{*} \\
& =\tau(y, g) \psi\left(x_{0}, g^{-1} \cdot y\right)^{*} \\
& =\psi(x, y)^{*} \tau\left(x_{0}, g^{-1}\right)^{*} .
\end{aligned}
$$

Hence $\phi=\psi$, implying that $P$ is the identity; a contradiction. So $\operatorname{ind}_{\tau}(H)$ is irreducible.

Corollary 2.2.5. If the multiplicity of the representation $\sigma$ in the restriction of $\operatorname{ind}_{\tau}(H)$ to $K$ is one then $\phi$ is $(G, K, V)$-zonal.

Proof. Set $P_{c}$ equal to $P_{0}$. 
Remark. In this context I should mention the very important result of Kobayashi [12]. With the notation as before suppose that $X$ has the structure of an $n$-dimensional complex manifold with quasi-invariant measure $\mu$. Form the holomorphic vector bundle $E$ over $G / K$ with fibre $V$ (this is the induced bundle). The representation $\sigma$ acts in the fibres and on the space of holomorphic $n$-forms on $G / K$ with values in $E$, it induces a representation $U$ of $G$. Kobayashi shows that there is a reproducing kernel $\phi$ for the Hilbert space $H$ of square integrable holomorphic $n$-forms. Furthermore whenever $H$ is non-trivial the restriction of $U$ to $H$ defines a unitary representation of $G$ so that $\phi$ satisfies an analogue of (2.2). Kobayashi then shows that this representation is always irreducible. In Section 3 we will see the idea behind Kobayashi's proof used in a particular example.

In the case where $\sigma$ is not irreducible it is more difficult to find conditions for zonality. In this direction we have the following result. Recall the definition of $H$, the kernel $\chi: G \times G \rightarrow B(V)$ [Eq. (2.3)] and the representation $W$ of $G$ defined by (2.6).

Proposition 2.2.6. If $P_{0}$ lies in the centre of the commuting algebra of the representation $k \rightarrow W_{k}$ of $K$ then the commuting algebra of $\left\{W_{g} \mid g \in G\right\}$ is isomorphic (as a von Neumann algebra) to the algebra of operators which commute with $\{\chi(g) \mid g \in G\}$.

The proof is not difficult and so we omit it. We conclude this subsection with the remark that there are close connections between the ideas discussed here and Naimark's notion of "quasihomogeneity" [13]. In fact when $\sigma$ is irreducible and $K$ is compact it is not difficult to show that $\operatorname{ind}_{\tau}(H)$ is quasihomogeneous in the sense of [13]. One should compare Proposition 2.2.6 with Naimark's Theorem 5.

\subsection{Functional Equations}

We lead into the connection between spherical functions and reproducing kernels with a brief introduction to Godement's work [5]. Suppose that $K$ is a compact subgroup of $G$, that $T$ is a representation of $G$ on a Banach space $B$ and that in the restriction of $T$ to $K, \sigma$ occurs with finite multiplicity. Denote by $B(\sigma)$ the closed subspace of $B$ formed by taking the linear span of those vectors which transform under the representation $k \rightarrow T_{k}$ of $K$ according to $\sigma$. If there exists a continuous projection $E(\sigma)$ of $B$ onto $B(\sigma)$ define

$$
\phi_{\sigma}(g)=\operatorname{Tr}\left(E(\sigma) T_{g}\right), \quad g \in G .
$$

Then $\phi_{\sigma}$ is called a spherical function for $G, K$ and Godement proves [5] that $\phi_{\sigma}$ satisfies

$$
\int_{K} \phi_{\sigma}\left(k g k^{-1} g^{\prime}\right) d k=\phi_{\sigma}(g) \phi_{\sigma}\left(g^{\prime}\right)
$$

if and only if $\sigma$ occurs once only in the representation $k \rightarrow T_{k}$ of $K$. Let 1 denote the trivial representation of $K$ and set

$$
T=\operatorname{ind}_{1}(H)
$$

where $H$ is a reproducing kernel Hilbert space with kernel $\phi$. 
Suppose that 1 occurs once only in $k \rightarrow T_{k}$ and set

$$
\chi_{x_{0}}(g)^{*}=\phi_{x_{0}}\left(g \cdot x_{0}\right)^{*}=\operatorname{Tr}\left(P_{0} T_{g^{-1}}\right) .
$$

So $\chi_{x_{0}}$ is a spherical function and the functional Equation (2.15) becomes:

$$
\int_{K} \phi_{x_{0}}\left(k g k^{-1} g^{\prime} \cdot x_{0}\right) d k=\phi_{x_{0}}\left(g \cdot x_{0}\right) \phi_{x_{0}}\left(g^{\prime} \cdot x_{0}\right) \text {. }
$$

Using (2.2) this reduces to:

$$
\int_{K} \phi_{x_{0}}\left(g^{\prime-1} \mathrm{~kg}^{-1} \cdot x_{0}\right)^{*} d k=\int T_{k^{-1}} \phi_{g^{\prime} \cdot x_{0}}\left(g^{-1} \cdot x_{0}\right)^{*} d k
$$

But $\int_{K} T_{k} d k$ is the projection $P_{1}$ onto the fixed point set of the representation $k \rightarrow T_{k}$ of $K$. So the functional equation is

$$
\left(P_{1} \phi_{x}^{*}\right)(y)=\phi_{x}\left(x_{0}\right)^{*} \phi_{x_{0}}(y)^{*}
$$

for all $x, y \in X$ (setting $g^{\prime} \cdot x_{0}=x$ and $g^{-1} \cdot x_{0}=y$ ).

Now remove the compactness assumption on $K$ and return to the general situation with the assumptions as in the preamble to Lemma 2.1.5. We impose, for the remainder of this section, the additional restriction that $\sigma$ be irreducible (and hence that the criterion for zonality given by Corollary 2.2.5 is valid). With $k \rightarrow S_{k}$ denoting the restriction to $K$ of $\operatorname{ind}_{\tau}(H)$ write $C(S)$ for the commuting algebra of $S$. By Lemma 2.1.5 there is a subspace of $H$, say $H(\sigma)$, which carries the representation $S^{\sim}$ of $K$ satisfying (see Mackey [11] for the terminology):

(i) $S$ is maximal primary.

(ii) Every subrepresentation of $S$ is quasiequivalent to $\sigma$.

[To see this one takes the central support in $C(S)$ of $P_{0}$.] As a consequence of Lemma 2.1.5 we have

Theorem 2.3.1. With the restrictions of the previous paragraph, the projection $P_{\sigma}$ onto $H(\sigma)$ satisfies for all $u \in V, x \in X$ :

$$
P_{\sigma} \phi_{x}^{*} u=\phi_{x_{0}}{ }^{*} \phi\left(x, x_{0}\right) * u
$$

if and only if the multiplicity of $\sigma$ in $\operatorname{ind}_{\tau}(H)$ restricted to $K$ is one.

In the particular case where $G$ is unimodular and $K$ is compact $P_{\sigma}$ has the form

$$
\left(P_{\sigma} F\right)(g)=\int_{K} \overline{C(k)} F\left(k^{-1} g\right) d k
$$

for $F \in H$, where $C(k)=d_{\sigma} \operatorname{Tr} \sigma(k)$ with $d_{\sigma}$ the dimension of $\sigma$ (see [5]). Recalling the definition of $\chi$ [Eq. (2.3)] we have

Corollary 2.3.2. With the assumptions above, the irreducible representation $\sigma$ occurs once only in the restriction of $\operatorname{ind}_{\tau}(H)$ to $K$ if and only if

$$
\int_{K} \overline{C(k)} \chi\left(g_{1}, k^{-1} g_{2}\right)^{*} d k=\chi_{e}\left(g_{2}\right)^{*} \chi_{g_{1}}(e)^{*} .
$$

Proof. Apply both sides of (2.18) to some $v \in V$ and then substitute from (2.3). The result then follows directly from Theorem 2.3.1.

When $V=\mathbb{C}$ we can interpret the theorem as asserting that the function $\chi_{e}$ is a positive definite spherical function of height one (see [5]). For, in this case

$$
\overline{c(k)} \chi_{g_{1}}\left(g_{2}\right)^{*}=\chi_{g_{1}}\left(g_{2} k\right)^{*}
$$


and so

$$
\int_{K} \chi_{e}\left(g_{1}^{-1} k^{-1} g_{2} k\right)^{*} d k=\chi_{e}\left(g_{2}\right)^{*} \chi_{g_{1}}(e)^{*} .
$$

Noting that a reproducing kernel is always a positive definite operator valued function [1], this suggests we consider the converse problem of when a positive definite spherical function is a reproducing kernel. Following Warner [14] we introduce the following assumptions and definitions.

(a) Let $G$ be unimodular with $K$ a large compact subgroup and let $\mu$ be a finite dimensional representation of $K$ acting on a Banach space $V$.

(b) Define a $\mu$-spherical function to be a continuous function

$\Psi: G \rightarrow \operatorname{Hom}(V, V)$

such that

$$
\Psi\left(k_{1} g k_{2}\right)=\mu\left(k_{1}\right) \Psi(g) \mu\left(k_{2}\right) .
$$

(c) Let $U$ be a topologically completely irreducible strongly continuous representation of $G$ in a Banach space $B$ such that in the restriction of $U$ to $K$, a given irreducible $\sigma$ occurs with multiplicity $m \neq 0$ (necessarily finite as $K$ is large in $G$ ). Let $\mu$ be the representation $m \sigma$ (i.e. the direct sum of $m$ copies of $\sigma$ ). Suppose further that there is a continuous projection $P_{\mu}$ onto the space of vectors in $B$ which transform according to $\sigma$ under $k \rightarrow U_{k}$ and define

$$
\Psi_{\sigma}^{U}(g)=P_{\mu} U_{g} P_{\mu} .
$$

Then $\Psi_{\sigma}^{U}$ is a $\mu$-spherical function. All spherical functions $\psi$, "of type $\sigma$ and height $m$ " (see [5]), have the form $\psi(g)=\operatorname{Tr} \Psi_{\sigma}^{U}(g)$ for some $\mu$-spherical function $\Psi_{\sigma}^{U}[14]$.

Now, on $V=P_{\mu} B$ we can define an inner product such that $\mu$ is unitary. Then $\psi$ defined by

$$
\psi(g)=\operatorname{Tr} \Psi_{\sigma}^{U}(g)
$$

is positive definite whenever $\Psi_{\sigma}^{U}$ is such that

$$
g \rightarrow\left\langle v, \Psi_{\sigma}^{U}(g) v\right\rangle_{V}
$$

is positive definite for all $v \in V$. In this case we call $\Psi_{\sigma}^{U}$ a positive definite $\mu$-spherical function. One may now use Theorem 1 of [1] to obtain a reproducing kernel Hilbert space for which

$$
g, g_{0} \rightarrow \Psi_{\sigma}^{U}\left(g^{-1} g_{0}\right)^{*}
$$

is the reproducing kernel. Alternatively consider the following interesting argument (due essentially to K.C. Hannabuss).

Define $\Psi_{\sigma}^{U}$ as above to be a positive definite $\mu$-spherical function. For convenience write $\Psi$ for $\Psi_{\sigma}^{U}$. Let $C_{0}(G, V)$ be the space of strongly continuous $V$-valued functions with compact support in $G$. Define:

$$
\Psi(f)\left(g_{0}\right)=\int_{G} \Psi\left(g^{-1} g_{0}\right) * f(g) d g
$$

for all $f \in C_{0}(G, V)$. Denote by $\Psi(G, V)$ the image of $C_{0}(G, V)$ under the map $f \rightarrow \Psi(f)$. On $\Psi(G, V)$ there is an inner product

$$
\langle\Psi(f), \Psi(f)\rangle_{\Psi}=\iint\left\langle f\left(g_{0}\right), \Psi\left(g^{-1} g_{0}\right)^{*} f^{\prime}(g)\right\rangle_{V} d g d g_{0} .
$$


Positivity of the inner product follows from the positive definite property of $\Psi$. If for some $f,\langle\Psi(f), \Psi(f)\rangle_{\Psi}=0$ then $\left\langle\Psi\left(f^{\prime}\right), \Psi(f)\right\rangle_{\Psi}=0$ for all $f^{\prime} \in C_{0}(G, V)$ by the Cauchy-Schwarz inequality. Equivalently,

$$
\int_{G}\left\langle f^{\prime}(g), \Psi(f)(g)\right\rangle_{V} d g=0
$$

for all $f^{\prime} \in C_{0}(G, V)$ and hence $\Psi(f)=0$. So $\langle,\rangle_{\Psi}$ is non-degenerate and we may complete $\Psi(G, V)$ to give a Hilbert space $H(\Psi)$ say.

Lemma 2.3.3. $H(\Psi)$ is a reproducing kernel Hilbert space with kernel $g, g_{0} \rightarrow$ $\Psi\left(g^{-1} g_{0}\right)^{*}$.

Proof. Let $\delta_{e}$ be the Dirac measure at the identity and as usual write $\Psi_{g}{ }^{*} v$ for the function $g_{0} \rightarrow \Psi\left(g^{-1} g_{0}\right)^{*} v(v \in V)$. Then by taking an approximate identity $\left\{f_{\alpha}\right\}_{\alpha \in A}$ for $G$ it is not difficult to see that $\Psi\left(f_{\alpha} v\right)$ converges weakly in $H(\Psi)$ to $\Psi\left(\delta_{e} v\right)$. Hence $\Psi_{g} v\left(g_{0}\right)^{*}=\Psi\left(\delta_{g} v\right)\left(g_{0}\right)$ and it is straightforward to verify that $g_{0}, g \rightarrow \Psi\left(g_{0}{ }^{-1} g\right)^{*}$ has the reproducing property for functions in $\Psi(G, V)$. An arbitrary element $F \in H(\Psi)$ may be identified with the function $F^{\sim}$ defined by $\left\langle v, F^{\top}(g)\right\rangle_{V}=\left\langle\Psi_{g}{ }^{*} v, F\right\rangle_{\Psi}$ (cf. Theorem 1 of [1]).

Returning to the main argument, observe that on $H(\Psi)$ we can define a strongly continuous unitary representation by

$$
W_{g} \Psi(f)\left(g^{\prime}\right)=\int_{G} \Psi\left(g_{0}{ }^{-1} g^{\prime}\right)^{*} f\left(g^{-1} g_{0}\right) d g_{0} .
$$

As the elements of $H(\Psi)$ satisfy $F(x k)=\mu\left(k^{-1}\right) F(x)$ the representation $g \rightarrow W_{g}$ is of the "induced" form we have been discussing to date.

Now let

(i) $V_{0}$ be the space of vectors $\left\{\Psi_{e}{ }^{*} v \mid v \in V\right\}$.

(ii) $P_{0}$ be the projection onto $V_{0}$ given by (Lemma 2.1.5)

$$
P_{0} \Psi_{g}^{*} v=\Psi_{e}^{*} \Psi(g, e)^{*} v \text {. }
$$

Then we have for all $u, v \in V$

$$
\left\langle\Psi_{e}^{*} u, P_{0} W_{g} P_{0} \Psi_{e}^{*} v\right\rangle=\left\langle u, \Psi(g, e)^{*} v\right\rangle
$$

so we have, from the definition of $\Psi=\Psi_{\sigma}^{U}$,

$$
\Psi_{e}(g)=E(\mu) U_{g} E(\mu)=P_{0} W_{g} P_{0} .
$$

Lemma 2.3.4. The representations $U$ and $W$ are Naimark equivalent (see [14]).

Proof. Let $B_{0}$ denote the dense subspace of $B$ spanned by $\left\{U_{g} v \mid g \in G, v \in V\right\}$. Let $H_{\Psi}$ be the span of $\left\{\Psi_{g}{ }^{*} v \mid g \in G, v \in V\right\}$. Following Kunze [1] define

$$
S U_{g} v=\Psi_{g}^{*} v \text {. }
$$

Extend $S$ linearly to $B_{0}$. Then clearly $S$ is one-one and onto $H_{\Psi}$ and satisfies $S U_{g}=W_{g} S$ on $B_{0}$. To complete the proof we will show that $S^{-1}: H_{\Psi} \rightarrow B_{0}$ is closable. For this, it is sufficient to show that if $\left\{F_{r}\right\} \subseteq H_{\Psi}$ is a sequence converging to zero in $H(\Psi)$ and $S^{-1} F_{r} \rightarrow f \in B_{0}$ then $f=0$.

If $F_{r}(g)=\left(\sum_{j} \Psi_{g_{j}}{ }^{*} v_{j}(g)\right)^{(r)}$ then, as norm convergence in $H(\Psi)$ implies pointwise convergence we have

$$
\left(\sum_{j} \Psi\left(g_{j}^{-1} g\right)^{*} v_{j}\right)^{(r)} \rightarrow 0 \text { for all } g \in G .
$$


That is, $E(\mu) U_{g^{-1}}\left(\sum_{j} U_{g_{j}} v_{j}\right)^{(r)} \rightarrow 0$ [using (2.20)], for all $g \in G$. Equivalently $E(\mu) U_{g} f=0$ for all $g \in G$. But $U$ is topologically completely irreducible and so $f$ must be zero.

We have now almost proved

Theorem 2.3.5. (i) If $\psi$ is a spherical function of type $\sigma$ and height $m$ defined via (2.19) in terms of a positive definite $\mu$-spherical $\Psi_{\sigma}^{U}$ (where $U$ is topologically completely irreducible and $\mu=m \sigma$ ) then there is a unitary irreducible representation $W$ of $G$ in a Hilbert space $H(\Psi)$ with reproducing kernel

$$
g, g_{0} \rightarrow \Psi_{\sigma}^{U}\left(g^{-1} g_{0}\right)^{*}
$$

such that $U$ is Naimark equivalent to $W$. Further, with $P_{0}$ denoting the projection onto the subspace of $H(\Psi)$ spanned by $\left\{\Psi_{e}^{*} v \mid v \in V\right\}$,

$$
\psi(g)=\operatorname{Tr}\left(P_{0} W_{g} P_{0}\right) .
$$

(ii) The reproducing kernel satisfies the functional equation

$$
\int_{K} \overline{C(k)} \Psi_{g_{0}}\left(k^{-1} g\right)^{*} d k=\Psi_{e}(g)^{*} \Psi_{g_{0}}(e)^{*} \text {. }
$$

Proof. (i) It remains only to prove that $W$ is irreducible. This follows from the remark on page 305 of [14].

(ii) If we can show that $P_{\sigma}=\mathrm{P}_{0}$ then the proof is complete. This follows from Proposition 4.5.1.6 of [14] (cf. also 6.1.1.8 of Volume 2 of [14].)

The theorem asserts the existence of a functional equation for positive definite $\mu$-spherical functions of arbitrary height. Note that only in the case where the height is one does there exist a corresponding equation for the spherical function

$$
\psi(g)=\operatorname{Tr} \Psi_{\sigma}^{U}(g) .
$$

Further, the reproducing kernel approach allows a reinterpretation of the functional equation as asserting (when the multiplicity of $\sigma$ is one) a sufficient condition for the irreducibility of the representation determined by the kernel.

\section{Application to $\mathrm{SU}(2,2)$}

\subsection{Notation}

Throughout this section $G=\mathrm{SU}(2,2)$ and $K=\mathrm{SU}(2) \times \mathrm{SU}(2) \times U(1)$ is the maximal compact subgroup of $G$. The notation and preliminary results are to be found in Ruhl [2]. The usual form of $\operatorname{SU}(2,2)$ consists of $4 \times 4$ matrices

$$
M=\left(\begin{array}{ll}
A & B \\
C & D
\end{array}\right)
$$

(where $A, B, C, D$ are $2 \times 2$ matrices over $\mathbb{C}$ ) such that

$$
M^{*} E=E M^{-1}, \quad \operatorname{det} M=1,
$$


with

$$
E=\left(\begin{array}{cc}
\sigma_{0} & 0 \\
0 & -\sigma_{0}
\end{array}\right)
$$

$\sigma_{0}$ being the $2 \times 2$ identity. We will consider the form of $\operatorname{SU}(2,2)$ obtained from that above by letting

$$
U=2^{-1 / 2}\left(\begin{array}{cc}
\sigma_{0} & -\sigma_{0} \\
\sigma_{0} & \sigma_{0}
\end{array}\right)
$$

and defining the map $M \rightarrow U M U^{-1}$.

This takes $\mathrm{SU}(2,2)$ onto the group of matrices of determinant one which preserve $\left(\begin{array}{cc}0 & \sigma_{0} \\ \sigma_{0} & 0\end{array}\right)$. In this realisation $G / K$ is naturally homeomorphic to the future tube [2]

$$
T=\left\{x+i y\left|x, y \in \mathbb{R}^{4}, y_{0}>\right| \boldsymbol{y} \mid\right\} .
$$

(Here we will use the usual notation of relativistic quantum mechanics.) SU $(2,2)$ acts on $T$ by

$$
\begin{aligned}
g \cdot W & =(R W+S)(T W+Q)^{-1} \\
& =\left(W T^{*}+Q^{*}\right)^{-1}\left(W R^{*}+S^{*}\right)
\end{aligned}
$$

where

$$
g=\left(\begin{array}{cc}
R & i S \\
-i T & Q
\end{array}\right)=U\left(\begin{array}{ll}
A & B \\
C & D
\end{array}\right) U^{-1}
$$

and $W=w_{0} \sigma_{0}+\boldsymbol{w} \cdot \boldsymbol{\sigma}\left(\boldsymbol{\sigma}=\left(\sigma_{1}, \sigma_{2}, \sigma_{3}\right)\right.$ being the Pauli matrices $)$.

The representations considered by Ruhl [2] are those of the holomorphic discrete series of SU (2,2) (see Harish-Chandra [15] and Graev [6] for the general theory). These may be regarded as representations induced from the compact Cartan subgroup of $\mathrm{SU}(2,2)$ or equally, from the maximal compact subgroup $K$ (cf. Langlands [7]). We will consider only a subclass of these representations.

Consider the Hilbert space $K_{n}$ whose elements are $\mathbb{C}$-valued functions holomorphic in $T$ and satisfying

$$
\|F\|_{n}^{2}=\int|F(W)|^{2}\left(y^{2}\right)^{n-4} d^{4} W<\infty
$$

where $y^{2}=y_{0}^{2}-y^{2}, d^{4} W$ is Lebesgue measure on $\mathbb{C}^{4}$ and $n \geqq 4$ is integral. Acting on this space is a unitary irreducible representation of $\operatorname{SU}(2,2)$ defined by

$$
\left(U_{g}^{n} F\right)(W)=\operatorname{det}\left(-T W^{*}+Q^{*}\right)^{-n} F\left(g^{-1} \cdot W\right)
$$

where

$$
g=\left(\begin{array}{cc}
R & i S \\
-i T & Q
\end{array}\right)
$$

The proof of this fact may be deduced from [6] using the results of [2]. We will give a proof, which depends on the reproducing kernel for $K_{n}$, at the end of Subsection 3.2 . 


\subsection{Limit Points}

We are interested in the cases $n=1,2,3$ treated briefly by Ruhl [2]. The case $n=3$ (but not $n=1,2$ ) is actually covered by the general theory of Knapp and Okamoto [16] so this analysis has some interest as a worked example. We follow the treatment by Gelfand et al. [17] of SU $(1,1)$ and introduce a new normalisation for the inner product. Define

$$
R_{n}(y)=\left(y^{2}\right)^{n-4} \theta\left(y_{0}\right) \theta\left(y^{2}\right) / \Gamma(n-2) \Gamma(n-3),
$$

where $\theta\left(y_{0}\right) \theta\left(y^{2}\right)$ is the characteristic function of $T$. Define

$$
\langle F, F\rangle_{n}=\int_{\mathbb{C}^{4}}|F(W)|^{2} R_{n}(y) d^{4} W .
$$

Recalling the definition of the generalised function $\left(y^{2}\right)_{+}^{\lambda}$ of Gelfand et al. [18] we see that

$$
R_{n}(y)=\theta\left(y_{0}\right)\left(y^{2}\right)_{+}^{n-4} / \Gamma(n-2) \Gamma(n-3) .
$$

It is in fact an analytic function of the index $n$, the factor $\Gamma(n-2) \Gamma(n-3)$ "cancelling" the poles of the numerator.

This generalized function takes the values

$$
\begin{aligned}
& R_{3}(y)=\delta_{+}\left(y^{2}\right)=\theta\left(y_{0}\right) \delta\left(y^{2}\right) \\
& R_{2}(y)=(\pi / 3) \delta(y) \\
& R_{1}(y)=(\pi / 4) \square \delta(y),
\end{aligned}
$$

for $n=3,2,1$ respectively. Substitution in (3.3) gives

$$
\|F\|_{3}^{2}=\int_{\mathbb{C}^{4}}|F(w)|^{2} \delta_{+}\left(y^{2}\right) d^{4} x d^{4} y
$$

where $w=x+i y \in \mathbb{C}^{4}$ and

$$
\|F\|_{2}^{2}=(\pi / 3) \int_{\mathbb{M}}|F(x)|^{2} d^{4} x
$$

where $\mathbb{M}=$ Minkowski space. As $\|F\|_{1}{ }^{2}$ is degenerate we will reserve the $n=1$ case until the next section.

From the work of Ruhl [2] one can deduce that there are Hilbert spaces $K_{3}, K_{2}$ of functions holomorphic on $T$ carring unitary representations of $\mathrm{SU}(2,2)$ given by (3.4) for $n=3,2$. One may also deduce that $K_{3}$ and $K_{2}$ have norms given by (3.5) and (3.6) respectively. I will give an independent treatment of these facts which illustrates some of the reproducing kernel techniques.

Let

$$
\partial T=\left\{x+i y \mid x, y \in \mathbb{R}^{4}, y^{2}=0, y_{0}>0\right\} .
$$

Consider the Hilbert spaces $L^{2}(\mathbb{M})$ and $L^{2}(\partial T, d v)$ where $d v$ is the measure $d^{4} x d^{3} y / 2 y_{0}$ on $\partial T$. Given $f \in L^{2}(\mathbb{M})$ and $f^{\prime} \in L^{2}(\partial T, d v)$, define functions $F$ and $F^{\prime}$ holomorphic in $T$ by

$$
\begin{aligned}
& F\left(w_{1}\right)=(\pi / 3) C_{2} \int_{\mathbb{M}} f(x)\left(\left(w_{1}-x\right)^{2}\right)^{-2} d^{4} x \\
& F^{\prime}\left(w_{1}\right)=C_{3} \int_{\mathbb{C}^{4}} f^{\prime}(w)\left(\left(w_{1}-w\right)^{2}\right)^{-3} d^{4} x d^{4} y \delta_{+}\left(y^{2}\right) .
\end{aligned}
$$


where $w=x+i y$ and $C_{2}$ and $C_{3}$ are normalisation constants. These integrals exist as the functions

$$
\begin{array}{ll}
x \rightarrow\left(\left(w_{1}-x\right)^{2}\right)^{-2}, & x \in \mathbb{M} \\
w \rightarrow\left(\left(w_{1}-\bar{w}\right)^{2}\right)^{-3}, & w \in \partial T
\end{array}
$$

lie in $L^{2}(\mathbb{M})$ and $L^{2}(\partial T, d v)$ respectively for all $w \in T$ (see below). We define function spaces $K_{2}$ and $K_{3}$ by (3.7) and (3.8) as the holomorphic extensions into $T$ of elements of $L^{2}(\mathbb{M})$ and $L^{2}(\partial T, d v)$. It remains to show that $K_{3}$ and $K_{2}$ are Hilbert spaces with norms given by (3.5) and (3.6). To do this we follow Ruhl [2] and use the Fourier-Laplace transform.

Let

$$
V_{p}^{+}=\left\{p \in \mathbb{R}^{4} \mid p_{0}>0, p^{2}>0\right\}
$$

and define Hilbert spaces $H_{\lambda}^{p}$ from functions $h: V_{p}^{+} \rightarrow \mathbb{C}$ satisfying

$$
(h, h)_{\lambda}=(\pi / 2) \int|h(p)|^{2} \theta\left(p_{0}\right) \theta\left(p^{2}\right) d^{4} p\left(p^{2}\right)^{-\lambda}<\infty
$$

where $\lambda \geqq 0$. Now define Hilbert spaces $H_{\lambda}^{w}$ of functions holomorphic in $T$ via the Fourier-Laplace transform $\mathscr{L}$ :

$$
(\mathscr{L} h)(w)=\int h(p) \exp (i p \cdot w) \theta\left(p_{0}\right) \theta\left(p^{2}\right) d^{4} p
$$

where $p \cdot w=p_{0} w_{0}-\boldsymbol{p} \cdot \boldsymbol{w}$. Observe that the functions $k_{w}^{\lambda}$ defined by

$$
k_{w}^{\lambda}(p)=\exp (-i p \cdot \bar{w})\left(p^{2}\right)^{\lambda}
$$

are in $H_{\lambda}^{p}$ for all $w \in T$. Hence (3.10) is defined as an ordinary integral being just the inner product $\left(k_{w}^{\lambda}, h\right)_{\lambda}$. Now Ruhl shows that $H_{n-2}^{w}=K_{n}$ for $n \geqq 4$. Further, the reproducing kernels for these spaces are defined by

$$
\begin{aligned}
\phi^{n}\left(w_{1}, w_{2}\right)^{*} & =\int \exp i p \cdot\left(w_{2}-\bar{w}_{1}\right)\left(p^{2}\right)^{n-2} d^{4} p \theta\left(p_{0}\right) \theta\left(p^{2}\right) \\
& =C_{n} \operatorname{det}\left(\frac{1}{2 i}\left(W_{2}-W_{1}{ }^{*}\right)\right)^{-n}
\end{aligned}
$$

where $C_{n}$ is a normalization factor (see [2]). The reproducing property follows from the relations

$$
\begin{aligned}
(\mathscr{L} h)(w) & =F(w)=\left(k_{w}^{n-2}, h\right)_{n-2} \\
& =\left\langle\mathscr{L} k_{w}^{n-2}, \mathscr{L} h\right\rangle_{n} \\
& =\left\langle\phi_{w}^{n *}, F\right\rangle_{n}, \quad F \in K_{n} .
\end{aligned}
$$

Now, for $n=2$ the above formulae still hold provided we define $\left\langle\phi_{w}^{2 *}, F\right\rangle_{2}$ by (3.7) (for a suitably chosen constant $C_{2}$ ). The relationship between $F$ and its "boundary value" $f$ in (3.7) is determined by the fact that if $F=\mathscr{L} h$ then $f$ is the Fourier transform of $h$. Further, the function $F_{y}$ defined by $F_{y}(x)=F(w), w=x+i y$ converges in the $L^{2}$ norm on $\mathbb{M}$ to $f$ as $y \rightarrow 0$ (see [2]).

When $n=3$ we need to do a little more work. Define a map $\mathscr{T}$ from $H_{1}^{p}$ into $L^{2}(\partial T, d v)$ by setting

$$
(\mathscr{T} f)(x+i y)=\int_{V_{p}^{+}} \exp i p \cdot(x+i y) f(p) d^{4} p, \quad x+i y \in \partial T,
$$


for those $f \in H_{1}^{p}$ which are integrable with respect to Lebesgue measure on $V_{p}^{+}$. $\mathscr{T}$ is norm preserving and so defines an isometry of $H_{1}^{p}$ onto a closed subspace of $L^{2}(\partial T, d v)$ (it is not difficult to show that $\mathscr{T}$ cannot map onto $L^{2}(\partial T, d v)$ ). Setting $f^{\prime}=\mathscr{T} h$ for $h \in H_{1}^{p}$ we see that

$$
\begin{aligned}
(\mathscr{L} h)(w) & =F^{\prime}(w)=\left(k_{w}^{1}, h\right)_{1} \\
& =\left\langle\mathscr{T} k_{w}^{1}, \mathscr{T} h\right\rangle_{3} \\
& =\left\langle\phi_{w}^{1 *}, f^{\prime}\right\rangle_{3} .
\end{aligned}
$$

That is, $K_{3}$ is just the image under $\mathscr{L}$ of $H_{1}^{p}$ and the norm (3.5) is well defined provided the "boundary value" $f^{\prime}$ of $F^{\prime}=\mathscr{L} h \in K_{3}$ is defined to be $\mathscr{T} h$.

We now derive proofs of unitarity and irreducibility of the representations $U^{n}$ defined by (3.4). The covariance relation (2.2) follows directly from the identity

$$
\left(g^{-1} \cdot W_{1}-g^{-1} \cdot W_{2}^{*}\right)=\left(W_{2}^{*} T^{*}+Q^{*}\right)^{-1}\left(W_{1}-W_{2}^{*}\right)\left(T W_{1}+Q\right)^{-1}
$$

where

$$
g^{-1}=\left(\begin{array}{cc}
R & i S \\
-i T & Q
\end{array}\right)
$$

using the definition (3.11) of $\phi^{n}$. Unitarity now follows from Theorem 1 of [1] (i.e. Theorem 2.1.3 of Subsection 2.1). Irreducibility may be obtained in several ways. The following argument may be found in Kobayashi's paper [12] and in Bargmann [3].

Let $\psi^{n *}$ be the kernel for some non-trivial invariant subspace of $K_{n}$, then $\psi^{n *}$ necessarily satisfies the covariance relation

$$
\psi^{n}\left(g^{-1} \cdot W_{1}, g^{-1} \cdot W_{2}\right)^{*}=\operatorname{det}\left[\left(W_{2}^{*} T^{*}+Q^{*}\right)\left(T W_{1}+Q\right)\right]^{n} \cdot \psi^{n}\left(W_{1}, W_{2}\right)^{*} .
$$

Since SU $(2,2)$ acts transitively on $T$ we can write

$$
\psi^{n}(W, W)=\operatorname{det}\left[\left(W^{*} T^{*}+Q^{*}\right)(T W+Q)\right]^{n} \psi^{n}\left(i \sigma_{0}, i \sigma_{0}\right)
$$

where $\left(\begin{array}{cc}R & i S \\ -i T & Q\end{array}\right)^{-1}$ takes $i \sigma_{0}$ to $W$. But equally $\phi^{n}(W, W)$ satisfies a similar relation and so

$$
\psi^{n}(W, W)=d_{n} \phi^{n}(W, W),
$$

for some $d_{n} \in \mathbb{R}$. Define a function $\chi^{n}$ by

$$
\chi^{n}\left(W_{1}, W_{2}\right)=d_{n} \phi^{n}\left(W_{1}, W_{2}\right)^{*}-\psi^{n}\left(W_{1}, W_{2}\right)^{*} .
$$

Make a change of variable by setting

$$
\zeta=W_{1}^{*}+W_{2}, \quad \eta=\left(W_{1}^{*}-W_{2}\right) / i .
$$

Then $\zeta, \eta \rightarrow \chi^{n}(\zeta, \eta)$ is holomorphic in $\zeta$ and $\eta$ and is zero when $W_{1}=W_{2}$. This is precisely when $\zeta, \eta$ are both real forcing $\chi^{n} \equiv 0$. Hence the representations $U^{n}$ are irreducible. 


\section{The Ladder Representations of SU(2, 2)}

\subsection{Preliminaries}

The ladder representations [8] are those which give rise to the conformal symmetry of zero mass particles. They have the interesting property that on restriction to a Poincaré subgroup of $S U(2,2)$ they remain irreducible [8]. Our intention in this section is to give a treatment of the ladder representations which makes use of the reproducing kernel techniques. We begin with some facts about the zero mass representations of the Poincare group (see [19] for an elaboration of the results listed here).

We will take the Poincare group $P$ to be $\mathbb{R}^{4} \subseteq S L(2, \mathbb{C})$. Write $\mathbb{P}^{4}$ for momentum space and let $X_{0}^{+}=\left\{p \in \mathbb{P}^{4} \mid p_{0}>0\right.$ and $\left.p^{2}=0\right\}$ be the surface of the forward light cone. The $P$ invariant measure on $X_{0}^{+}$is

$$
d \mu_{0}(p)=\delta_{+}\left(p^{2}\right) d^{4} p=\theta\left(p_{0}\right) \delta\left(p^{2}\right) d^{4} p .
$$

Let $V_{s}$ be the $(2 s+1)$-dimensional Hilbert space carrying the representation $\sigma_{s}$ of $\operatorname{SL}(2, \mathbb{C})$ labelled $(s, 0)$ (see [19] for this labelling) where the norm is chosen so that $\sigma_{s}$ restricted to $\mathrm{SU}(2) \subseteq \mathrm{SL}(2, \mathbb{C})$ is unitary. Let $d \mu_{s}$ be the measure

$$
d^{3} \boldsymbol{p} / 2|\boldsymbol{p}|^{2 s+1} \equiv p_{0}^{-2 s} \delta_{+}\left(p^{2}\right) d^{4} p
$$

on $X_{0}^{+}$and define a non-unitary representation $N$ of $P$ on $L^{2}\left(X_{0}^{+}, V_{s}, d \mu_{s}\right)$ by

$$
\left(N_{g} f\right)(p)=\sigma_{s}(\Lambda) \exp (i n \cdot p) f\left(\Lambda^{-1} \cdot p\right)
$$

where $g=(n, \Lambda) \in P$.

I will take the Lie algebra of $\operatorname{SL}(2, \mathbb{C})$ to be spanned by $\left\{J_{i}, L_{i} \mid i=1,2,3\right\}$ with

$$
\begin{aligned}
& {\left[J_{i}, J_{j}\right]=i \varepsilon_{i j k} J_{k} ; \quad\left[L_{i}, L_{j}\right]=-i \varepsilon_{i j k} J_{k}} \\
& {\left[J_{i}, L_{j}\right]=i \varepsilon_{i j k} L_{k} .}
\end{aligned}
$$

Denote by $p^{\wedge}$ the element $(1,0,0,1) \in \mathbb{P}^{4}$ and let $\chi_{s}\left(p^{\wedge}\right)$ be the unique vector of norm one in $V_{s}$ such that

$$
\sigma_{s}\left(\exp \left(i \lambda J_{3}\right)\right) \chi_{s}\left(p^{\wedge}\right)=\exp (i \lambda s) \chi_{s}\left(p^{\wedge}\right) .
$$

If $p$ is any point on $X_{0}{ }^{+}$let

$$
l_{p}: \hat{p} \rightarrow\left(p_{0}, 0,0, p_{0}\right)
$$

be the $\operatorname{SL}(2, \mathbb{C})$ matrix

$$
\left(\begin{array}{cc}
p_{0}{ }^{1 / 2} & 0 \\
0 & p_{0}{ }^{-1 / 2}
\end{array}\right)
$$

and $R_{p}$ be the rotation taking $\left(p_{0}, 0,0, p_{0}\right)$ to $\left(p_{0}, p_{1}, p_{2}, p_{3}\right)$. Putting the above together we define

$$
\chi_{s}(p)=\sigma_{s}\left(R_{p} l_{p}\right) \chi_{s}\left(p^{\wedge}\right) .
$$

Now, the zero mass (positive energy) helicity $s$ representation of $P(s=0$, $1 / 2,1,3 / 2, \ldots)$ is constructed on the subspace $\Gamma_{s}$ of $L^{2}\left(X_{0}{ }^{+}, V_{s}, d \mu_{s}\right)$ consisting 
of solutions of the equation:

$$
\sum_{i=1}^{3} \dot{\sigma}_{s}\left(J_{i}\right) p_{i} f(p)=s p_{0} f(p)
$$

where $\dot{\sigma}_{s}$ denotes the representation of the Lie algebra of SL $(2, \mathbb{C})$ corresponding to $\sigma_{s}$. A straightforward calculation shows that the non-orthogonal projection onto $\Gamma_{s}$ is given by

$$
(P f)(p)=\sigma_{s}\left(R_{p} l_{p}\right) P_{0} \sigma_{s}\left(l_{p}^{-1} R_{p}^{-1}\right) f(p),
$$

where $P_{0}$ is the projection in $V_{s}$ defined by

$$
P_{0} v=\left\langle\chi_{s}\left(p^{\wedge}\right), v\right\rangle_{V_{s}} \chi_{s}\left(p^{\wedge}\right), \quad v \in V_{s} .
$$

We will write

$$
E(p)=\sigma_{s}\left(R_{p} l_{p}\right) P_{0} \sigma_{s}\left(l_{p}^{-1} R_{p}^{-1}\right) .
$$

The advantage of defining the Poincare representations on $L^{2}\left(X_{0}{ }^{+}, V_{s}, d \mu_{s}\right)$ is that the inner product may be transformed onto Minkowski space. To see this we introduce first the Schwarz space on $\mathbb{R}^{4}\left(\mathbb{P}^{4}\right)$ of $C^{\infty}$ functions of fast decrease taking values in $V_{s}: \mathscr{S}\left(\mathbb{R}^{4}, V_{s}\right)\left(\mathscr{S}\left(\mathbb{P}^{4}, V_{s}\right)\right)$. Let $\mathscr{S}_{0}\left(\mathbb{P}^{4}, V_{s}\right)$ be the subspace of $\mathscr{S}\left(\mathbb{P}^{4}, V_{s}\right)$ consisting of functions of compact support not containing the origin. Write $\mathscr{F}$ for the Fourier transform from both $L^{2}\left(\mathbb{P}^{4}, V_{s}\right)$ to $L^{2}\left(\mathbb{R}^{4}, V_{s}\right)$ and from $\mathscr{S}^{\prime}\left(\mathbb{P}^{4}, V_{s}\right)$ to $\mathscr{S}^{\prime}\left(\mathbb{R}^{4}, V_{s}\right)$. Finally, note that every function $f \in L^{2}\left(X_{0}{ }^{+}, V_{s}, d \mu_{s}\right)$ defines a distribution $f \delta_{+}$on $\mathscr{S}\left(\mathbb{P}^{4}, V_{s}\right)$ by

$$
\left(f \delta_{+}\right)(\phi)=\int_{X_{0}^{+}} \sum_{v=1}^{2 s+1} f_{v}(\omega, \boldsymbol{p}) \phi_{v}(\omega, \boldsymbol{p}) d^{3} \boldsymbol{p} / 2 \omega
$$

where $\omega=|\boldsymbol{p}|$.

Now, it is not hard to show that the inner product in $L^{2}\left(X_{0}{ }^{+}, V_{s}, d \mu_{s}\right)$ transforms, for functions $\phi, \psi \in \mathscr{F} \mathscr{S}_{0}\left(\mathbb{P}^{4}, V_{s}\right)$ into:

$$
\begin{aligned}
\langle\phi, \psi\rangle_{0}^{s}= & i \int_{x_{0}=0}\left[\frac{\partial}{\partial x_{0}}\left(\beta_{s} * \phi^{+}\right)(x)^{*} \cdot\left(\beta_{s} * \psi^{-}\right)(x)\right. \\
& \left.-\left(\beta_{s} * \phi^{+}\right)(x)^{*} \frac{\partial}{\partial x_{0}}\left(\beta_{s} * \psi^{+}\right)(x)\right] d^{3} x
\end{aligned}
$$

where

(i) $\phi^{+}=\mathscr{F}\left(\phi \delta_{+}\right)=(\mathscr{F} \phi) *\left(\mathscr{F} \delta_{+}\right)$,

(ii) $\beta_{s}$ is the Fourier transform of the tempered distribution

$$
\left.p_{0}^{-s} \equiv(1 / 2)\left(p_{0}+i 0\right)+(1 / 2)\left(p_{0}-i 0\right)\right)^{-s}
$$

using the notation of Gelfand et al. [18],

(iii) $\beta_{s} * \phi^{+}$denotes convolution in the $x_{0}$ variable only. (Note that it is not trivial that $\beta_{s} * \phi^{+}$is well defined.)

Now (4.6) is an integral over the spacelike hypersurface $x_{0}=0$. If we write $\langle,\rangle_{\Sigma}^{s}$ for the corresponding integral over any sufficiently smooth spacelike hypersurface $\Sigma$, an application of Green's theorem gives

$$
\langle,\rangle_{\Sigma}^{s}=\langle,\rangle_{0}^{s} \text {. }
$$


Essentially what we are doing here is taking a space of $V_{s}$-valued solutions of $\square F(x)=0$ and defining an inner product by an integral over a Cauchy initial data surface. The form $\langle,\rangle_{\Sigma}^{s}$ is positive definite because we are restricting to positive energy solutions of the wave equation. This space of solutions $\mathscr{F} \mathscr{S}_{0}\left(\mathbb{P}^{4}, V_{s}\right)$ may now be completed to give a Hilbert space $H_{s}$ say, whose elements may be identified with those of $\mathscr{T} L^{2}\left(X_{0}{ }^{+}, V_{s}, d \mu_{s}\right)$ where $\mathscr{T}$ is the map

$$
f \rightarrow \mathscr{F}\left(f \delta_{+}\right), \quad f \in L^{2}\left(X_{0}{ }^{+}, V_{s}, d \mu_{s}\right) .
$$

\subsection{Some Properties of $\Gamma_{s}$}

If $f \in \Gamma_{s}$ and $g=\left(0, R_{p} l_{p}\right)^{-1} \in P$ then we have, from Equation (4.3)

$$
\sum_{i} \dot{\sigma}_{s}\left(J_{i}\right) p_{i}\left(N_{g} \cdot f\right)\left(p^{\wedge}\right)=s\left(N_{g} \cdot f\right)\left(p^{\wedge}\right),
$$

and this reduces to

$$
\dot{\sigma}_{s}\left(J_{3}\right)\left(N_{g} \cdot f\right)\left(p^{\wedge}\right)=s\left(N_{g} \cdot f\right)\left(p^{\wedge}\right) .
$$

Recall that $\chi_{s}\left(p^{\prime}\right)$ is the unique vector of norm one satisfying this last equation. Hence

$$
\left(N_{g} \cdot f\right)\left(p^{\wedge}\right)=c_{g} \chi_{s}\left(p^{\wedge}\right)
$$

where $c_{g} \in \mathbb{C}$ depends only on $p$. Thus we may define a measurable function $f^{0}: X_{0}{ }^{+} \rightarrow \mathbb{C}$ by $f^{0}(p)=c_{g}$ where $g=\left(0, R_{p} l_{p}\right)^{-1}$. We now have

$$
\begin{aligned}
f(p) & =\sigma_{s}\left(R_{p} l_{p}\right)\left(N_{g} \cdot f\right)\left(p^{\wedge}\right) \\
& =\sigma_{s}\left(R_{p} l_{p}\right) c_{g} \chi_{s}\left(p^{\wedge}\right) \\
& =f^{0}(p) \chi_{s}(p) .
\end{aligned}
$$

Our object is to show that $f^{0} \in \Gamma_{0}=L^{2}\left(X_{0}{ }^{+}, d \mu_{0}\right)$.

The first step is to note that with

$$
L_{3}=\left(\begin{array}{cc}
i / 2 & 0 \\
0 & -i / 2
\end{array}\right)
$$

we have $l_{p}=\exp \left(-i \log \left(p_{0}\right) L_{3}\right)$. Secondly, recall that in the representation $(s, 0)[19]$

$$
i \dot{\sigma}_{s}\left(J_{3}\right)=\dot{\sigma}_{s}\left(L_{3}\right)
$$

and therefore

$$
\dot{\sigma}_{s}\left(l_{p}\right) \chi_{s}\left(p^{\wedge}\right)=p_{0}^{s} \chi_{s}\left(p^{\wedge}\right) .
$$

Finally since $\sigma_{s}\left(R_{p}\right)$ is unitary

$$
\begin{aligned}
\|f\|^{2} & =\int\left|f^{0}(p)\right|^{2} \chi_{s}\left(p^{\wedge}\right)^{*} \chi_{s}\left(p^{\wedge}\right) \delta_{+}\left(p^{2}\right) d^{4} p \\
& =\int\left|f^{0}(p)\right|^{2} \delta_{+}\left(p^{2}\right) d^{4} p<\infty .
\end{aligned}
$$

This proves

Lemma 4.2.1. Every function $f \in \Gamma_{s}$ has the form

$$
f(p)=f^{0}(p) \chi_{s}(p)
$$


for some $f^{0} \in \Gamma_{0}$. Furthermore the inner product of two functions in $\Gamma_{s}$ is given by

$$
\left\langle f_{1}, f_{2}\right\rangle=\int_{\mathbb{P}^{4}} \overline{f_{1}^{0}(p)} f_{2}^{0}(p) \delta_{+}\left(p^{2}\right) d^{4} p
$$

so that the map $f^{0} \rightarrow f$ from $\Gamma_{0}$ onto $\Gamma_{2}$ is an isometry.

Let $f_{j}(j=1,2)$ be elements of $\Gamma_{s}$ and denote by $F_{j}^{0}$ the Fourier transform of $f_{j}^{0} \delta_{+}$. Then the inner product has the simple form:

$$
\left\langle\mathscr{T} f_{1}, \mathscr{T} f_{2}\right\rangle_{\Sigma}^{s}=i \int_{\Sigma}\left[\partial_{\mu} \overline{F_{1}^{0}(x)} F_{2}^{0}(x)-\overline{F_{2}^{0}(x)} \partial_{\mu} F_{1}^{0}(x)\right] \cdot d \sigma^{\mu}(x)
$$

(with $\partial_{\mu}=\frac{\partial}{\partial x^{\mu}}$ and $d \sigma^{\mu} \equiv$ "area measure" in $\Sigma$ ) whenever $f_{j}^{0} \delta_{+}$is actually the product of a function $f_{j}^{0}$ in $\mathscr{S}_{0}\left(\mathbb{P}^{4}, \mathbb{C}\right)$ with the distribution $\delta_{+}$.

We conclude this subsection by determining some dense subspaces of $\Gamma_{s}$ and $L^{2}\left(X_{0}^{+}, V_{s}, d \mu_{s}\right)$.

Lemma 4.2.2. Define for each $w \in T, G_{w}{ }^{-}(p)=\exp (-i p \cdot \bar{w})$. The functions ${G_{w}}^{-} \in \Gamma_{0}$ for all $w \in T$ and their linear span is dense.

Proof.

$$
\left\|G_{w}{ }^{-}\right\|^{2}=\int \exp (-2 p \cdot y) \delta_{+}\left(p^{2}\right) d^{4} p
$$

where $y$ is the imaginary part of $w$. Hence

$$
\left\|G_{w}{ }^{-}\right\|^{2}=\pi / 2\left(y_{0}^{2}-|\boldsymbol{y}|^{2}\right)\left\langle\infty \text { since } y_{0}\right\rangle|\boldsymbol{y}| \text { in } T \text {. }
$$

Density follows from two known results:

(i) the functions $\left\{\omega \rightarrow \exp \left(-2 \omega y_{0}\right)\left|y_{0}>\right| \boldsymbol{y} \mid, \boldsymbol{y}\right.$ fixed $\}$ span a dense subspace of $L^{2}(0, \infty)$;

(ii) the functions $\left\{\boldsymbol{p} \rightarrow \exp (i \boldsymbol{p} \cdot \boldsymbol{x}) \mid \boldsymbol{x} \in \mathbb{R}^{3}\right\}$ span a dense subspace of the space of functions square integrable on the sphere of radius $\omega=|\boldsymbol{p}|$ (the measure being that arising from $\left.d^{3} p / 2 \omega\right)$. As a corollary we note that the functions $f_{w}$ defined by $f_{w}(p)=G_{w}{ }^{-}(p) \chi_{s}(p)$ span a dense subspace of $\Gamma_{s}$. A similar argument to that above gives

Lemma 4.2.3. The functions $\overline{h_{w}^{v}}: p \rightarrow p_{0}^{2 s} \overline{G_{w}(p)} e_{v}$ where $w$ ranges over $T$ and

$$
e_{v}(v=1, \ldots, 2 s+1)
$$

ranges over an orthonormal basis of $V_{s}$, span a dense subspace of $L^{2}\left(X_{0}{ }^{+}, V_{s}, d \mu_{\mathrm{s}}\right)$.

\subsection{Hilbert Spaces of Holomorphic Functions}

This is the central topic of this section. Here we produce the Hilbert spaces in which the ladder representations act, as Hilbert spaces of holomorphic functions and exhibit the reproducing kernels.

Let $f: X_{0}{ }^{+} \rightarrow V_{s}$ be square integrable with respect to $d \mu_{s}$, then its FourierLaplace transform defined componentwise by

$$
F_{v}(w)=\int \exp (i p \cdot w) f_{v}(p) \delta_{+}\left(p^{2}\right) d^{4} p
$$


exists as an ordinary integral by Lemma 4.2 .3 and the fact that (4.8) is just

$$
F_{v}(w)=\left\langle h_{w}{ }^{v}, f\right\rangle \text {. }
$$

Furthermore, $f_{v} \delta_{+}$is a tempered distribution with support in the closure of $V_{p}^{+}\left[V_{p}^{+}\right.$defined by (3.9)] implying that (4.8) defines a function holomorphic in $T$ [2]. Denoting the Fourier-Laplace transform by $\mathscr{L}$ define

$$
H_{s}^{w}=\mathscr{L} L^{2}\left(X_{0}{ }^{+}, V_{s}, d \mu_{s}\right)
$$

as the Hilbert space with inner product

$$
\left\langle F_{1}, F_{2}\right\rangle_{s}=\int f_{1}(p)^{*} f_{2}(p) \delta_{+}\left(p^{2}\right) d^{4} p / p_{0}^{2 s}
$$

where $F_{j}=\mathscr{L}\left(f_{j} \delta_{+}\right) j=1,2$.

The first question we will settle is: what can be said about the Shilov boundary values of elements of $H_{s}^{w}$ (Minkowski space is the Shilov boundary of $T$ [21]).

We know [20] that such functions will converge in a distribution theoretic sense to their boundary values $\mathscr{T} f=\mathscr{F}\left(f \delta_{+}\right)$but in fact more is true.

Theorem 4.3.1. With $F=\mathscr{L} f$ and $F_{y}$ defined by

$$
\begin{aligned}
& F_{y}(x)=F(w) ; \quad w=x+i y, \\
& \lim _{y \rightarrow 0}\left\|F_{y}-\mathscr{T} f\right\|_{\Sigma}^{s}=0 .
\end{aligned}
$$

So $F$ converges in norm to its boundary value $\mathscr{T} f$.

Proof. Firstly, $\mathscr{T} f$ is an element of $H_{s}$ and for each $y$ in $V_{+}$where

$$
V_{+}=\left\{y \in \mathbb{R}^{4}\left|y_{0}>\right| \boldsymbol{y} \mid\right\},
$$

the function $F_{y}$ is in $H_{s}$. Secondly, since $F_{y}$ is the image under $\mathscr{T}$ of the function

$$
p \rightarrow \exp (-p \cdot y) f(p),
$$

we have

$$
\left\|F_{y}\right\|_{s}^{2}=\int_{\mathbb{P}^{4}} \exp (-2 p \cdot y)\|f(p)\|_{V_{s}}^{2} \delta_{+}\left(p^{2}\right) d^{4} p / p_{0}^{2 s} .
$$

Thus

$$
\left\|F_{y}-\mathscr{T} f\right\|_{\Sigma}^{s}=\int_{\mathbb{P}^{4}}\|(\exp (-p \cdot y)-1) f(p)\|_{V_{s}} \delta_{+}\left(p^{2}\right) d^{4} p / p_{0}^{2 s} .
$$

But

$$
|\exp (-p \cdot y)-1|^{2}\|f(p)\|^{2} / p_{0}^{2 s} \leqq\|f(p)\|^{2} / p_{0}^{2 s}
$$

and

$$
|\exp (-p \cdot y)-1|^{2}\|f(p)\|^{2} / p_{0}^{2 s} \rightarrow 0 \quad \text { as } \quad y \rightarrow 0 .
$$

Therefore by the Lebesgue dominated convergence theorem,

$$
\lim _{y \rightarrow 0}\left\|F_{y}-\mathscr{T} f\right\|_{\Sigma}^{s}=0 \text {. }
$$


Next observe that $H_{s}^{w}$ is a reproducing kernel Hilbert space with kernel

$$
\begin{aligned}
\phi^{s}\left(w_{1}, w_{2}\right)^{*} & =\int \exp \left(i p \cdot\left(w_{2}-\bar{w}_{1}\right)\right) p_{0}^{2 s} \delta_{+}\left(p^{2}\right) d^{4} p \\
& =\left\langle h_{w_{2}}^{-v}, h_{w_{1}}^{-v}\right\rangle \cdot I
\end{aligned}
$$

where $I$ is the identity operator on $V_{s}$. This is clear from

$$
\left\langle\phi_{w_{1}}^{s} * e_{v}, F\right\rangle \stackrel{\text { def }}{=} \int \exp \left(i p \cdot w_{1}\right) f_{v}(p) \delta_{+}\left(p^{2}\right) d^{4} p=F_{v}\left(w_{1}\right) .
$$

\section{Define}

$$
\psi\left(w_{1}, w_{2}\right)^{*}=\left\langle h_{w_{2}}-h_{w_{1}}\right\rangle .
$$

From the known Laplace transform of $\delta_{+}\left(p^{2}\right)$ we have

$$
\psi\left(w_{1}, w_{2}\right)^{*}=\pi / 2\left(w_{2}-\bar{w}_{1}\right)^{2},
$$

whence for $s=0$ we have recovered the reproducing kernel for the $n=1$ case of Section 3.

Proposition 4.3.2. The space $\Gamma_{s}^{w}=\mathscr{L} \Gamma_{s}$ is a reproducing kernel Hilbert space with an operator-valued kernel whose components are

$$
\begin{aligned}
\Phi_{\mu \nu}\left(w_{1}, w_{2}\right)^{*} & =\left\langle e_{\mu}, \Phi\left(w_{1}, w_{2}\right)^{*} e_{v}\right\rangle_{V_{s}} \\
& =\left\langle e_{\mu} \int \exp \left[i p \cdot\left(w_{2}-\bar{w}_{1}\right)\right] p_{0}^{2 s} E(p)^{*} e_{\nu}\right\rangle_{V_{s}} \delta_{+}\left(p^{2}\right) d^{4} p .
\end{aligned}
$$

Proof. We prove firstly that $\Phi_{w}{ }^{*} u \in \Gamma_{s}^{w}$ for all $u \in V_{s}$. Recalling the definition of $E(p)$ by (4.4) we have

$$
p_{0}^{2 s} E(p)^{*} u=\left\langle\chi_{s}(p), u\right\rangle \chi_{s}(p)
$$

and hence $\Phi_{w}{ }^{*} u$ lies in $\Gamma_{s}^{w}$ provided the function

$$
p \rightarrow \exp (-i p \cdot \bar{w})\left\langle\chi_{s}(p), u\right\rangle
$$

is in $L^{2}\left(X_{0}{ }^{+}, d \mu_{0}\right)$ (Lemma 4.2.1). But this last function has norm

$$
\begin{aligned}
& \int \exp (-2 p \cdot y)\left|\left\langle\chi_{s}(p), u\right\rangle\right|^{2} \delta_{+}\left(p^{2}\right) d^{4} p \\
& =\|u\|^{2} \int \exp (-2 p \cdot y) p_{0}^{2 s} \delta_{+}\left(p^{2}\right) d^{4} p<\infty .
\end{aligned}
$$

It remains to check the reproducing property. Given $F \in \Gamma_{s}^{w}$ we have

$$
F(w)=\int \exp (i p \cdot w) f^{0}(p) \chi_{s}(p) \delta_{+}\left(p^{2}\right) d^{4} p
$$

and therefore

$$
\begin{aligned}
\left\langle\Phi_{w}{ }^{*} u, F\right\rangle_{s} & =\int \exp (i p \cdot w)\left\langle E(p)^{*} u, \chi_{s}(p)\right\rangle f^{0}(p) \delta_{+}\left(p^{2}\right) d^{4} p \\
& =\int \exp (i p \cdot w)\left\langle u, \chi_{s}(p)\right\rangle f^{0}(p) \delta_{+}\left(p^{2}\right) d^{4} p
\end{aligned}
$$

by the definition of $\chi_{s}(p)$ and $E(p)$. So

$$
\left\langle\Phi_{w}{ }^{*} u, F\right\rangle_{s}=\langle u, F(w)\rangle_{V_{s}}
$$

as required. 
Using the results of Subsection 4.1 we can rewrite the above formulae in Minkowski space terms. Let $f \in L^{2}\left(X_{0}{ }^{+}, V_{s}, d \mu_{s}\right)$ then for $F=\mathscr{L}\left(f \delta_{+}\right)$

$$
\begin{aligned}
F_{v}(w) & =\left\langle\phi_{w}^{s *} e_{v}, F\right\rangle_{\Sigma}^{s} \\
& =\int \exp (i p \cdot w) f_{v}(p) \delta_{+}\left(p^{2}\right) d^{4} p \\
& =\left\langle G_{w}^{-}, f_{v}\right\rangle .
\end{aligned}
$$

Now $\left(\mathscr{T} G_{w}\right)(x)^{*}=\pi / 2(w-x)^{2}$ and so for $f$ "sufficiently well behaved",

$$
\begin{aligned}
F_{v}(w) & =\left\langle G_{w}^{-}, f_{v}\right\rangle=\left\langle\mathscr{T} G_{w}^{-}, \mathscr{T} f_{v}\right\rangle_{0} \\
& =\frac{i \pi}{2} \int_{x_{0}=0}\left[\frac{\partial}{\partial x_{0}}\left(1 /(w-x)^{2}\right) \mathscr{T} f_{v}(x)-\frac{1}{(w-x)^{2}} \frac{\partial}{\partial x_{0}}\left(\mathscr{T} f_{v}(x)\right)\right] d^{3} \boldsymbol{x} \\
& \equiv \frac{i \pi}{2} \int_{\Sigma}\left[\partial_{\mu}\left(1 /(w-x)^{2}\right) \mathscr{T} f_{v}(x)-\frac{1}{(w-x)^{2}} \partial_{\mu}\left(\mathscr{T} f_{v}(x)\right)\right] d \sigma^{\mu}(x) .
\end{aligned}
$$

These are just the classical Kirchoff formulae (expressing the solution $\mathscr{T} f_{v}$ of the wave equation in terms of its initial data via the Green's function for positive energy solutions), extended to functions holomorphic in $T$. These formulas have a distribution theoretic meaning for arbitrary $f \in L^{2}\left(X_{0}{ }^{+}, V_{s} d \mu\right)$ [22]. Thus the reproducing property of the kernel merely expresses the "reproducing property" of the Green's function (cf. [23]). Similar formulae can be derived for elements of $\Gamma_{s}^{w}$.

Finally, we note that from (2.12) there exist bounds for the elements of $\Gamma_{s}^{w}$ and $H_{s}^{w}$. For $H_{s}^{w}$ we compute $\left(F=\mathscr{L}\left(f \delta_{+}\right)\right)$,

$$
\left|F_{v}(w)\right|^{2} \leqq\left\|h_{w}{ }^{-}\right\|^{2}\|f\|^{2}=(-1 / 2)^{2 s} \frac{\partial^{2 s}}{\partial y_{0}^{2 s}}\left(1 / y^{2}\right)\|f\|^{2}
$$

where $y$ is the imaginary part of $w$. For $\Gamma_{s}^{w}$ the function $w \rightarrow \Phi(w, w)^{*}$ can be determined after some calculation as

$$
\Phi(w, w)_{\mu \nu}^{*}=\left[\pi(2 s) ! \delta_{\mu \nu} /\left(2 y^{2}\right)^{2 s+1}\right]\left(\sigma_{s}\right)_{\mu \nu}\left(\begin{array}{cc}
y_{0}+|\boldsymbol{y}| & 0 \\
0 & y_{0}-|\boldsymbol{y}|
\end{array}\right)
$$

where $\left(\sigma_{s}\right)_{\mu \nu}$ denotes the $\mu v^{\text {th }}$ matrix element. This gives a bound by substitution in

$$
\left|F_{v}(w)\right| \leqq \Phi(w, w)_{v v}^{1 / 2}\|F\| .
$$

Remark. Let $D$ be the domain of $\left(-\nabla^{2}\right)^{1 / 2}$ in $L^{2}\left(\mathbb{R}^{3}\right)$. Then the elements of $\Gamma_{0}^{w}$ are the holomorphic extensions into $T$ of tempered distributions on Minkowski space defined via the Kirchoff formula from initial data in $D$.

\subsection{The Ladder Representations}

Consider the following action of $\mathrm{SU}(2,2)$ on $V_{s}$-valued functions holomorphic in $T$ :

$$
(g \cdot F)(W)=\operatorname{det}(T W+Q)^{-1-2 s} \sigma_{s}\left(W T^{*}+Q^{*}\right) F\left(g^{-1} \cdot W\right)
$$


where

(i) $W=w_{0} \sigma_{0}+\boldsymbol{w} \cdot \boldsymbol{\sigma}$ and $g^{-1}=\left(\begin{array}{cc}R & i S \\ -i T & Q\end{array}\right)$,

(ii) $g^{-1} \cdot W=(R W+S)(T W+Q)^{-1}$,

(iii) $\sigma_{s}$ is extended in the obvious way to $\operatorname{GL}(2, \mathbb{C})$.

A distinguished Poincaré subgroup of $\mathrm{SU}(2,2)$ is given by matrices with $T=0$, i.e. matrices of the form

$$
g=\left(\begin{array}{cc}
a & i V a^{*-1} \\
0 & a^{*-1}
\end{array}\right) ; \quad g^{-1}=\left(\begin{array}{cc}
a^{-1} & -i a^{-1} V \\
0 & a^{*}
\end{array}\right)
$$

where $a \in \operatorname{SL}(2, \mathbb{C})$ and $V=v_{0} \sigma_{0}+\boldsymbol{v} \cdot \boldsymbol{\sigma}, v_{\mu} \in \mathbb{R}$. Restricting (4.10) to Poincaré elements we have

$$
(g \cdot F)(W)=\sigma_{s}(a) F\left(a^{-1}(W-V) a^{*-1}\right) .
$$

This is just the representation $N$ defined by (4.1) transferred via the Laplace transform onto functions in $T$. Restricting therefore to $\Gamma_{s}^{w}$ we obtain the (positive energy) zero mass helicity $s$ representation of the Poincaré group.

Now it is not hard to compute the expressions for the generators of $\operatorname{SU}(2,2)$ acting on the functions holomorphic in T. Setting the imaginary part of $w$ equal to zero in these expressions yields the formal expressions of Mack and Todorov [8] for the infinitesmal form of the ladder representations acting on functions on Minkowski space. It would be nice to have a global proof of the unitarity of these representations but unfortunately only the $s=0$ case is easily treated. The proof is as follows.

The reproducing kernel is

$$
\phi^{0}\left(W_{1}, W_{2}\right)^{*}=\frac{\pi}{2} \operatorname{det}\left(\frac{1}{2 i}\left(W_{2}-W_{1}^{*}\right)\right)^{-1}
$$

and the covariance relation for

$$
g^{-1}=\left(\begin{array}{cc}
R & i S \\
-i T & Q
\end{array}\right)
$$

is just

$$
\phi^{0}\left(g^{-1} W_{1}, g^{-1} \cdot W_{2}\right)^{*}=\operatorname{det}\left[\left(W_{1}^{*} T^{*}+Q^{*}\right)\left(T W_{2}+Q\right)\right] \cdot \phi^{0}\left(W_{1}, W_{2}\right)^{*} .
$$

But this is no more than the covariance relation (2.2). So by Theorem 1 of [1] we have unitarity of the representation (4.10) for $s=0$.

Note that this proof fails for the higher spin cases simply because it is not clear that these are of the "induced form" that we have been discussing (cf. Gross [24]). One can prove however that $(g \cdot F)(W)=0$ for all $g \in G$ if and only if

$$
\sum_{i} \sigma_{s}\left(J_{i}\right) \frac{\partial}{\partial w_{i}} F(W)=s \frac{\partial}{\partial w_{0}} F(W) .
$$

So $H_{s}^{w}$ is not invariant under the $\mathrm{SU}(2,2)$ action (cf. [25]). 
Acknowledgement. Most of the work described here was done at the Mathematical Institute, Oxford under the supervision of Dr. K. C. Hannabuss whose assistance and encouragement I am pleased to acknowledge. I would also like to thank Mr. M. A. Gerzon and Dr. E. B. Davies for some helpful discussions. The paper was completed with the financial support of a Rothman's Fellowship.

\section{References}

1. Kunze, R.A.: In: Proc. of the Conference in Functional Analysis (ed. B. R. Gelbaum). Irvine: California Univ. 1966

2. Ruhl, W.: Commun. math. Phys. 27, 53 (1972); 30, 237 (1973); 34, 149 (1973)

3. Bargmann, V.: Comm. Pure Appl. Math. 14, 187 (1961)

4. Perelomov, A. M.: Commun. math. Phys. 26, 222 (1972)

5. Godement, R.: Trans. Amer. Math. Soc. 73, 496 (1952)

6. Graev, M.I.: Dokl. Akad. Nauk. SSSR 98, 517 (1954)

7. Langlands, R.P.: Amer. J. Math. 85, 99 (1963)

8. Mack, G., Todorov, I.: J. Math. Phys. 10, 2073 (1969)

9. Krein, M. G.: Amer. Math. Soc. Transl. (2) 34, 69-108, 109-164 (1963)

10. Varadarajan, V.S.: Geometry of quantum theory, Vol. II. New York: Van Nostrand 1970

11. Mackey, G. W.: The theory of group representations, Lecture Notes, Department of Mathematics, Univ. of Chicago, 1955

12. Kobayashi, S.: Proc. Amer. Math. Soc. 12, 359 (1961); J. Math. Soc. Japan 20, 638 (1968)

13. Naimark, M.A.: Dokl. Akad. Nauk SSSR 205, 1040 (1972)

14. Warner, G.: Harmonic analysis on semisimple Lie groups, Vols. I. II. Berlin-Heidelberg-New York: Springer 1972

15. Harish-Chandra: Amer. J. Math. 77, 743 (1955); 78, 1 (1956); 78, 564 (1956)

16. Knapp, A.W., Okamoto, K.: J. Funct. Anal. 9, 375 (1972)

17. Gelfand,I.M., Graev, M.I., Vilenkin, N.Ya.: Generalised functions, Vol. 5. New York: Academic Press 1966

18. Gelfand,I.M., Shilov, G.E.: Generalised functions, Vol. I. New York: Academic Press 1966

19. Niederer, U., O’Raifeartaigh, L.: Fortschr. Phys. 22, 111 (1974); 22, 131 (1974)

20. Streater, R.F., Wightman, A.S.: PCT, spin and statistics and all that. New York: W. A. Benjamin, Inc. 1964

21. Stein,E.M., Weiss, G.L.: Introduction to fourier analysis on Euclidean spaces. Princeton: Princeton Univ. Press 1971

22. Vladimirov, V.S.: Methods of the theory of functions of many complex variables. Cambridge Mass.: M.I.T. Press 1966

23. Bergman, S., Schiffer, M.: Kernel functions and elliptic differential equations in mathematical physics. New York: Academic Press 1953

24. Gross, L.: J. Math. Phys. 5, 687 (1964)

25. Muggli, J.: Helv. Phys. Acta 46, 253 (1973)

Communicated by H. Araki

Received October 11, 1975 
